\title{
Structure of a human insulin peptide-HLA-DQ8 complex and susceptibility to type 1 diabetes
}

K. H. Lee et al.

Nature Immunology 2, 501-507 (2001).

In the June issue of Nature Immunology, the Protein Data Bank accession number for the insulin peptide-HLA-DQ8 structure was missing. The 